\title{
Correlations between Visual Recognition Memory and Neocortical and Hippocampal Glucose Metabolism after Bilateral Rhinal Cortex Lesions in the Baboon: Implications for Alzheimer's Disease
}

\author{
Xavier Blaizot, ${ }^{1,2}$ Kenichi Meguro, ${ }^{1}$ Isabelle Millien, ${ }^{1,2,3}$ Jean Claude Baron, ${ }^{1,4}$ and Chantal Chavoix ${ }^{1,3}$ \\ 1 Institut National de la Santé et de la Recherche Médicale Unit 320, ${ }^{2}$ Commissariat à l'Energie Atomique Direction des

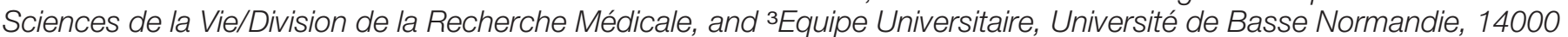 \\ Caen, France, and ${ }^{4}$ Department of Neurology, University of Cambridge CB2 2QQ, Cambridge, UK
}

In Alzheimer's disease (AD), the rhinal cortex is the area earliest and most affected by neurofibrillary tangles, and the degree of temporoparietal glucose hypometabolism and rhinal cortex atrophy are both correlated with dementia severity. In monkeys, damage to the rhinal cortex leads to severe impairment in declarative memory, which is also affected preferentially in early $A D$. To investigate the contribution of rhinal alterations to the interrelationships between cerebral hypometabolism and declarative memory impairment observed in $A D$, we studied the effects of excitotoxic bilateral rhinal lesions in baboons on cerebral glucose consumption (CMRglc) as measured by positron emission tomography and performance on a visual recognition memory task as assessed in parallel by a delayed nonmatching-to-sample task. We reported previously that these rhinal lesions induce both a long-lasting hypometabolism in several remote brain regions (Meguro et al., 1999) and impaired memory performance (Chavoix et al., 2002). The present analysis indicates that across lesioned and sham baboons, memory scores were significantly positively correlated ( $p<$ 0.05; Spearman) with concomitant CMRglc values of several brain areas, such as neocortical associative and posterior hippocampal regions. These findings, reminiscent of those reported in $A D$, suggest that the neurodegenerative process that affects the rhinal cortex in early $A D$ plays a crucial role in the pattern of brain hypometabolism and consequently in the declarative memory impairments characteristic of this disease.

Key words: Alzheimer's disease; cerebral glucose metabolism; declarative memory; entorhinal and perirhinal cortices; excitotoxic lesion; nonhuman primate
Alzheimer's disease (AD) has characteristic neuropathological, clinical, and metabolic features, but their interrelationships are still unclear. Significant correlations have been reported between declarative memory impairment and declines in cerebral glucose consumption (CMRglc) in the hippocampal region (i.e., hippocampal formation plus parahippocampal gyrus), thalamus and cingulate, and prefrontal and posterior associative cortices (Perani et al., 1993; Meltzer et al., 1996; Desgranges et al., 1998), pointing to dysfunction in widespread neuronal networks. Furthermore, atrophy of the hippocampal region, and especially of the rhinal $(\mathrm{Rh})$ cortex [which comprises the entorhinal $(\mathrm{ERh})$ and perirhinal (PRh) cortices], the area affected earliest and most severely by the neurofibrillary tangles (Braak and Braak, 1991), is correlated with both the degree of dementia (Juottonen et al., 1998; Bobinski et al., 1999) and hypometabolism in the tem-

Received May 31, 2002; revised Aug. 12, 2002; accepted Aug. 14, 2002.

This work was supported by the Institut National de la Santé et de la Recherche Medicale, the Commissariat à l'Energie Atomique, the Région Basse Normandie, the University of Caen, and by the Fondation pour la Recherche Médicale and the Fondation France Alzheimer. We thank Claude Le Mestric for valuable contribution to the PET studies and Karim Benali, Françoise Chapon, and Brigitte Landeau for helpful comments in the data analysis.

Correspondence should be addressed to Dr. Chantal Chavoix, Equipe Universitaire, Université de Basse Normandie, Centre Hospitalier Universitaire de Caen, Service de Neurologie Déjerine, 14000 Caen, France. E-mail: chavoix-c@chucaen.fr.

X. Blaizot's present address: Human Neuroanatomy Laboratory, School of Medicine, University of Castilla-La Mancha, 02071 Albacete, Spain.

K. Meguro's present address: Section of Neuropsychology, School of Medicine, Tohoku University, Sendai 980-8575, Japan.

Copyright (C) 2002 Society for Neuroscience $0270-6474 / 02 / 229166-05 \$ 15.00 / 0$ poroparietal areas (Yamaguchi et al., 1997; Meguro et al., 2001). Interestingly, the Rh cortex, which is strongly interconnected with both the neocortex and the hippocampus (Van Hoesen and Pandya, 1975; Insausti et al., 1987a; Suzuki and Amaral, 1994; Insausti and Munoz, 2001), plays a major role in declarative memory, as shown by lesion (Meunier et al., 1993; Murray, 1996; Malkova et al., 2001; Chavoix et al., 2002) and recent activation (Blaizot et al., 2000; Davachi and Goldman-Rakic, 2001) studies in the nonhuman primate.

It has been suggested that in $\mathrm{AD}$, both the brain hypometabolism and the memory impairment could be accounted for at least partially by disconnection of the hippocampal/rhinal complex (Mielke et al., 1996). Thus far, however, no study has attempted to relate these two features of AD with the neuropathological damage in the rhinal cortex. This is probably because of both the complexities involved and the difficulties in accounting for the histopathology already present in other structures, such as the hippocampus and neocortical regions.

To elucidate this issue and specifically to investigate whether $\mathrm{Rh}$ cortex lesions would mimic early AD in terms of both memory impairment and CMRglc declines, we developed a baboon model involving severe $\mathrm{Rh}$ cortex neuronal loss induced by excitotoxic lesion. We reported previously that this lesion leads to severe and sustained impairment in declarative memory (Chavoix et al., 2002) and significant CMRglc decreases in several areas, such as the posterior hippocampal region [including the hippocampal formation and the surrounding parahippocampal cortex (areas $\mathrm{TH}$ and $\mathrm{TF}$ )] and the inferior parietal, posterior temporal, pos- 


\section{Intended Lesion}
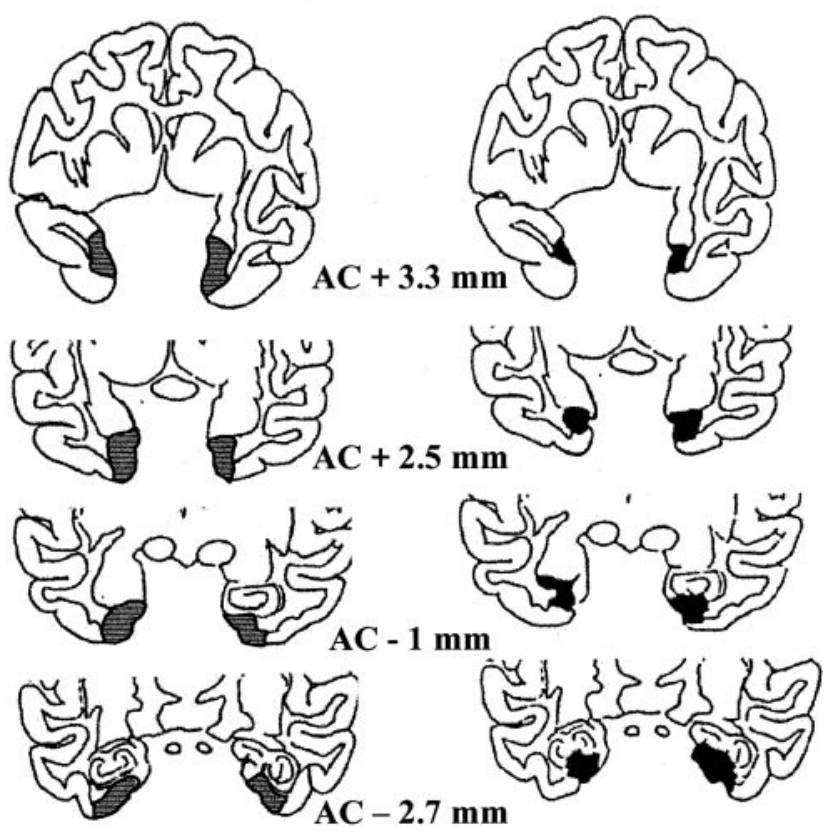

Figure 1. Schematic damage in baboon RH4 compared with intended lesion (gray areas). Shown in black are the areas with $>25 \%$ neuronal loss. Approximate rostrocaudal distance (in millimeters) from the anterior commissure $(A C)$ is indicated below each coronal section.

terior cingulate, and association occipital cortices (Meguro et al., 1999), as seen in AD. Interestingly, the CMRglc decrease in several areas was correlated positively with the extent of rhinal damage (Meguro et al., 1999). It was unclear, however, whether these CMRglc reductions in brain areas connected anatomically with the damaged Rh cortex were causative to the memory impairment. To address this issue, we have now assessed, in a partly overlapping sample of baboons with and without Rh cortex lesions, the relationships between performance in visual recognition memory as reported by Chavoix et al. (2002) and the CMRglc in a selective set of brain areas known to be involved in visual recognition memory and/or affected metabolically in AD (i.e., the hippocampal, temporal, parietal, dorsolateral prefrontal, and posterior cingulate regions, and the thalamus).

\section{MATERIALS AND METHODS}

Subjects and general considerations. Eight male young adult baboons (Papio anubis), weighing 10-15 kg at the beginning of the experiment, were used in this study; six were part of the group reported by Meguro et al. (1999) (RH1, RH3, RH4, SH2, SH3, and SH4, see below); two were new. At the start of memory training, the animals were experimentally naive. After preoperative CMRglc measurements and memory assessment, the ERh and $\mathrm{PRh}$ cortices were lesioned neurotoxically in four animals (called RH1, RH3, RH4, and RH5); the four remaining baboons served as sham-operated controls (called SH2, SH3, SH4, and SH5).

Histological damage in these eight baboons has been described in detail previously (Blaizot et al., 1999; Meguro et al., 1999). Briefly, severe neuronal loss in both the ERh and PRh cortices was found in all RH baboons, with a mean individual percentage cell loss in the $\mathrm{Rh}$ cortex ranging from 50 to $75 \%$ on both sides. Baboons RH1 and RH4 showed the best rhinal lesions, with minimal extra damage (for illustration, see Fig. 1). In baboons RH3 and RH5, the most rostral part of the Rh cortex was slightly damaged. The neighboring areas were essentially spared, although partial inadvertent damage was found, especially in the hippocampus of baboons RH3 and RH5. No significant damage was observed in SH baboons.

Surgery. This protocol was approved both by the Institut National de la
Santé et de la Recherche Médicale (INSERM) Bureau of Animal Experimentation and by the INSERM Neurosciences Board. The surgical and magnetic resonance (MR) procedures have been described in detail previously (Blaizot et al., 1999). Briefly, bilateral lesioning of the Rh cortex was made using a novel methodology that combines MR techniques and cranial landmarks to predetermine the target sites, taking into account the cerebral vessel locations, with stereotaxic neurosurgery. The lesions were made by local injections (22 target sites per side) of ibotenic acid $(1.2 \mu \mathrm{l}$ at $\mathrm{pH} 7.4 ; 12-15 \mu \mathrm{g} / \mu \mathrm{l}$ in PBS). The sham-operated baboons underwent the same surgical procedure, except that the needles were stopped $3 \mathrm{~mm}$ above the $\mathrm{Rh}$ cortex without any injection.

Behavioral testing. Testing was conducted in a sound-proof, dimmed room equipped with a computerized test apparatus adapted from that used by Mishkin's group (National Institute of Mental Health, Bethesda, MD).

Visual recognition memory was assessed using a computerized visual delayed nonmatching-to-sample (DNMS) task with trial-unique stimuli that requires the animal to choose, from two meaningless drawings, the one it did not see before. Before surgery, the baboons were trained and tested on a delay subtest of the task. One to 2 months after surgery (range, 34-62 d), after successful retraining and retesting on the delay subtest, the baboons were assessed on the list-length subtest of the DNMS, in which lists of $1,3,6,10$, or 15 drawings were presented before the choice tests in mixed order and for 5 consecutive days. In the present study of declarative memory, we used performance on the list-lengths 3 and 6, because the list-length 1 reflects rule learning, and performance on the list-lengths 10 and 15 were at chance level for both groups (for detailed methodology and results, see Chavoix et al., 2002).

CMRglc measurements. As described in detail by Le Mestric et al. (1993, 1998) and Meguro et al. (1999), positron emission tomography (PET) was performed using the ${ }^{18}$ F-fluoro-2-deoxy-D-glucose (FDG) method and a LETI TTV03 device (seven slices; intrinsic resolution, $5.5 \times 5.5 \times 9 \mathrm{~mm}, x, y, z)$; after ${ }^{68} \mathrm{Ge}$ transmission, FDG uptake images were obtained in the coronal plane (50-60 min after injection) under light dissociative anesthesia (phencyclidine; $\mathrm{N}_{2} \mathrm{O} / \mathrm{O}_{2}$ at a ratio of 2 to 1 ). Briefly, FDG images were transformed into parametric CMRglc (in milligrams per $100 \mathrm{gm}$ per minute), and the CMRglc data set was sampled in eight geometrical regions of interest (ROIs) (see Tables 1 and 2 for complete list) defined on six individual coregistered MRI cuts. These ROIs were selected for their assumed involvement in declarative memory and/or their metabolic alteration in AD. Each baboon underwent sequential postsurgical PET (Meguro et al., 1999). The CMRglc values used for the correlations with memory scores were those from the PET study performed closest in time to the cognitive assessment (from 25 to $50 \mathrm{~d}$ after surgery).

Data analysis. The correlations between the CMRglc values for each of the eight ROIs (averaged for both sides) and memory scores (averaged percentage of correct responses on list-lengths 3 and 6) were tested with Spearman's nonparametric test.

\section{RESULTS}

Table 1 shows the DNMS performance and the CMRglc values in the eight regions used for the correlational analysis for each baboon. Note that (1) most RH baboons performed at chance level with lists of stimuli (i.e., $<60 \%$ correct responses), and (2) the lowest CMRglc values were generally found in baboons RH3 and RH4 (i.e., those with the most extensive rhinal damage).

Table 2 shows that performance on the DNMS list-length subtest was significantly positively correlated (up to $p<0.02$ ) with CMRglc values in five of the eight regions assessed on the basis of a priori hypotheses, namely, the inferior parietal, posterior hippocampal, posterior temporal, dorsolateral prefrontal, and posterior cingulate regions. Figure 2 shows illustrative scatterplots for the posterior hippocampal, inferior parietal, and prefrontal dorsolateral regions.

\section{DISCUSSION}

We found significant positive correlations (i.e., in the neurobiologically expected direction) between declarative memory performance and CMRglc in five of the eight brain regions known to be important in visual recognition memory and/or affected metabol- 
Table 1. Individual DNMS performance and CMRglc values in the lesioned $(\mathrm{RH} ; n=4)$ and sham-operated $(\mathrm{SH} ; n=4)$ groups

\begin{tabular}{|c|c|c|c|c|c|c|c|c|}
\hline & \multicolumn{8}{|c|}{ Animal } \\
\hline & RH1 & RH3 & RH4 & RH5 & $\mathrm{SH} 2$ & SH3 & SH4 & SH5 \\
\hline DNMS performance & 52 & 59 & 53 & 61 & 61 & 62 & 64 & 65 \\
\hline \multicolumn{9}{|l|}{ CMRglc values } \\
\hline Dorsolateral prefrontal & 7.4 & 7.6 & 7.6 & 12.2 & 7.5 & 13.3 & 12.6 & 12.9 \\
\hline Inferior parietal & 6.4 & 6.6 & 5.3 & 8.2 & 7.2 & 10.4 & 9.2 & 9.1 \\
\hline Anterior temporal & 6.1 & 5.1 & 5.5 & 7.5 & 5.0 & 8.2 & 9.1 & 9.2 \\
\hline Posterior temporal & 5.5 & 5.2 & 4.3 & 8.3 & 6.5 & 8.9 & 9.5 & 6.7 \\
\hline Posterior cingulate & 6.1 & 7.0 & 6.3 & 11.2 & 7.1 & 10.9 & 10.8 & 11.0 \\
\hline Anterior hippocampal & 4.4 & 3.2 & 5.0 & 4.7 & 4.3 & 6.6 & 7.3 & 7.3 \\
\hline Posterior hippocampal & 3.8 & 3.8 & 4.4 & 5.5 & 4.6 & 5.8 & 6.7 & 7.3 \\
\hline Thalamus & 5.9 & 5.1 & 5.6 & 10.3 & 8.5 & 10.1 & 12.3 & 10.2 \\
\hline
\end{tabular}

Performance is expressed in percentage of correct responses and CMRglc in milligrams per $100 \mathrm{mg}$ per minute.

\begin{tabular}{|c|c|c|}
\hline ROIs & $\rho$ & $p$ \\
\hline Dorsolateral prefrontal & 0.762 & $0.044^{*}$ \\
\hline Inferior parietal & 0.857 & $0.023^{*}$ \\
\hline Anterior temporal & 0.667 & 0.078 \\
\hline Posterior temporal & 0.738 & $0.050^{*}$ \\
\hline Posterior cingulate & 0.738 & $0.050^{*}$ \\
\hline Anterior hippocampal & 0.690 & 0.068 \\
\hline Posterior hippocampal $^{a}$ & 0.905 & $0.017^{*}$ \\
\hline Thalamus & 0.714 & 0.059 \\
\hline
\end{tabular}

${ }^{a}$ Includes the posterior part of the hippocampus and the posterior parahippocampal cortex.

*Significant correlation $(p \leq 0.05)$.

ically in AD. Thus, the CMRglc decline observed in this limbicneocortical network in the lesioned baboons appears to subtend their visual recognition memory impairment at least in part. This in turn suggests that the early neuropathological changes that affect the Rh cortex in AD may be responsible for the synaptic dysfunction in this network, and that this synaptic dysfunction is involved in the declarative memory deficits characteristic of this disorder.

Before we discuss further our findings with respect to the rhinal cortex and AD, some methodological issues need to be addressed. First, the correlations were run on the data from four lesioned and four sham-operated baboons (i.e., both groups contributed to the significant correlations observed). Thus, a relationship between memory performance and CMRglc values seems to exist even within the control animals (Fig. 2), which were included to enlarge the sample size and enhance the variance in the data. This is in agreement with the correlations reported between memory performance and resting-state metabolism across aging in healthy human subjects (Eustache et al., 1995). Second, to limit the risk of false-positives and false-negatives caused by our relatively small sample size, we used nonparametric correlations throughout, which lie on the conservative side. Third, the resting CMRglc measurements were performed under light dissociative anesthesia, in contrast to studies in conscious AD subjects. However, the effects of this anesthetic regimen in low doses on brain metabolism are assumed to be negligible (Fitch et al., 1978).

Our a priori hypotheses about which brain regions would be

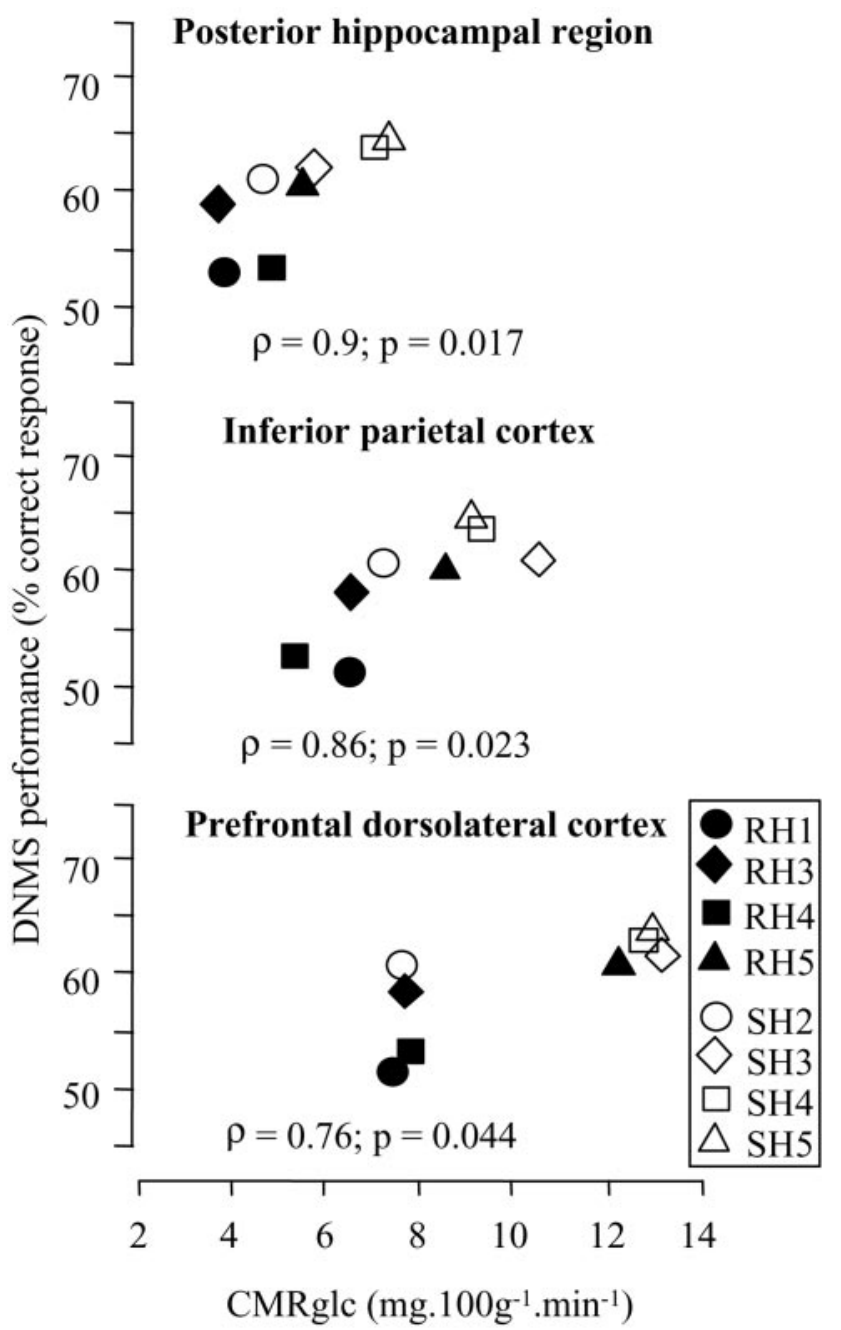

Figure 2. Illustration of the correlations between CMRglc and performance on the DNMS task for the posterior hippocampal, inferior parietal, and prefrontal dorsolateral regions $[n=8$ baboons (4 lesioned and 4 sham-operated) for each correlation]. The correlation coefficients (Spearman's $\rho$ ) and corresponding $p$ (two-tailed) values are shown for each correlation.

correlated with DNMS performance were based on their implications in $\mathrm{AD}$ and/or in visual recognition memory (see the introductory remarks). Among this set of selected regions, our significant memory-CMRglc correlations concerned the poste- 
rior hippocampal region and the posterior cingulate, inferior parietal, posterior temporal, and prefrontal dorsolateral regions. A trend of correlation, also in the expected direction, was found in the remaining three regions [i.e., the thalamus $(p=0.059)$, anterior hippocampal ( $p=0.068)$, and anterior temporal ( $p=$ 0.078 ) regions]. Similar findings have been reported consistently in $\mathrm{AD}$, although most studies concerned verbal declarative memory, in contrast to visual declarative memory in the present study. Consistent with our results, however, significant correlations have been reported in mild-to-moderate AD between visual declarative memory scores and resting CMRglc or cerebral blood flow in the hippocampal region (Perani et al., 1993; Ohnishi et al., 1995; Ishii et al., 1996), posterior parietal cortex (O'Brien et al., 1992; Soininen et al., 1995; Meltzer et al., 1996), temporal cortex (Soininen et al., 1995; Sabbagh et al., 1997), and posterior cingulate cortex (Desgranges et al., 1998). Concerning the hippocampal region, our finding is also consistent with the decreased activation in the left hippocampus and parahippocampal gyrus observed in patients with AD compared with elderly controls during visual memory encoding using functional MRI (Rombouts et al., 2000).

Our findings are also in agreement with lesional studies in monkeys that show the implications of the hippocampal formation (Alvarez et al., 1995) and thalamus (Aggleton and Mishkin, 1983; Zola-Morgan and Squire, 1985) in visual recognition memory, these two regions being connected directly with the $\mathrm{Rh}$ cortex (Insausti et al., 1987a,b; Suzuki, 1996). That our correlations did not quite reach significance for the anterior hippocampal and anterior temporal regions, two structures also related to visual memory, is somewhat surprising. It should be noted, however, that (1) the rostral rhinal cortex, whose main connection is with the anterior hippocampal region (here including the anterior hippocampal formation and parts of the amygdaloid complex), was relatively spared from damage (Blaizot et al., 1999) and (2) partial volume effects may account for the weak correlation for the anterior temporal region, because this region encompassed both the superior and inferior temporal cortices, but only the latter has strong rhinal connections.

Finally, the visuospatial working memory processes required in our task, because of both the shape and complexity of the stimuli and interferences between encoding and recognition of series of stimuli, could explain the correlation seen with the inferior parietal and dorsolateral prefrontal cortices. Both regions have been shown to be involved in visuospatial working memory in the monkey (Goldman-Rakic, 1987; Chafee and Goldman-Rakic, 1998), and correlations between performance on visuospatial short-term memory tasks and metabolic activity in the inferior parietal and dorsolateral prefrontal cortices have been reported in patients with AD (Perani et al., 1993; Desgranges et al., 1998). Interestingly, performance on the visuospatial span in AD was also correlated significantly with resting CMRglc in the thalamus (Desgranges et al., 1998).

Although it could be argued that similar correlations between memory performance and CMRglc might occur after lesioning of any other part of this cognitive network, it is likely that the more the damaged region is involved in the functional network (which would apply to the Rh cortex and visual recognition memory), the stronger the relationships with the cognitive performance. Although this hypothesis should be tested further, it has been shown, for instance, that damage to the cholinergic structures of the basal forebrain, known to play a modulatory role only in visual recognition memory, impairs DNMS performance only slightly (Aigner et al., 1991) and accordingly has marginal effects at best on CMRglc (Le Mestric et al., 1998).

In conclusion, our findings from memory-CMRglc correlations are in large part consistent with earlier findings in AD. They suggest that (1) the early and severe neuronal loss in the Rh cortex that characterizes this neurodegenerative disorder plays an important part in both the declarative memory impairment and the hippocampal-neocortical hypometabolism typical of AD and (2) the latter are interrelated, so that the memory impairment in AD may reflect, at least in its early stages, functional disruption of a distributed large-scale network resulting from rhinal disconnections that may be amenable to therapeutic intervention, such as local neuron transplantation.

\section{REFERENCES}

Aggleton JP, Mishkin M (1983) Visual recognition impairment following medial thalamic lesions in monkeys. Neuropsychologia 21:189-197.

Aigner TG, Mitchell SJ, Aggleton JP, DeLong MR, Struble RG, Price DL, Wenk GL, Pettigrew KD, Mishkin M (1991) Transient impairment of recognition memory following ibotenic-acid lesions of the basal forebrain in macaques. Exp Brain Res 86:18-26.

Alvarez P, Zola-Morgan S, Squire LR (1995) Damage limited to the hippocampal region produces long-lasting memory impairment in monkeys. J Neurosci 15:3796-3807.

Blaizot X, Meguro K, Le Mestric C, Constans JM, Luet D, Baron JC, Chavoix C (1999) Combined use of T1-weighted MRI and MRA for stereotaxic lesioning of the nonhuman primate brain: application to the rhinal cortex. Exp Brain Res 126:31-40.

Blaizot X, Landeau B, Baron JC, Chavoix C (2000) Mapping the visual recognition memory network with PET in the behaving baboon. J Cereb Blood Flow Metab 20:213-219.

Bobinski M, de Leon MJ, Convit A, De Santi S, Wegiel J, Tarshish CY, Saint LL, Wisniewski HM (1999) MRI of entorhinal cortex in mild Alzheimer's disease. Lancet 353:38-40.

Braak H, Braak E (1991) Neuropathological stageing of Alzheimerrelated changes. Acta Neuropathol 82:239-259.

Chafee MV, Goldman-Rakic PS (1998) Matching patterns of activity in primate prefrontal area $8 \mathrm{a}$ and parietal area 7ip neurons during a spatial working memory task. J Neurophysiol 79:2919-2940.

Chavoix C, Blaizot X, Meguro K, Landeau B, Baron JC (2002) Excitotoxic lesions of the rhinal cortex in the baboon differentially affect visual recognition memory, habit memory and spatial executive functions. Eur J Neurosci 15:1225-1236.

Davachi L, Goldman-Rakic PS (2001) Primate rhinal cortex participates in both visual recognition and working memory tasks: functional mapping with 2-DG. J Neurophysiol 85:2590-2601.

Desgranges B, Baron JC, de la Sayette V, Petit-Taboue MC, Benali K, Landeau B, Lechevalier B, Eustache F (1998) The neural substrates of memory systems impairment in Alzheimer's disease: a PET study of resting brain glucose utilization. Brain 121:611-631.

Eustache F, Rioux P, Desgranges B, Marchal G, Petit-Taboue MC, Dary M, Lechevalier B, Baron JC (1995) Healthy aging, memory subsystems and regional cerebral oxygen consumption. Neuropsychologia 33: 867-887.

Fitch W, MacGeorge AP, MacKenzie ET (1978) Anesthesia for studies of cerebral circulation: a comparison of phencyclidine and althesin in the baboon. Br J Anaesth 50:985-991.

Goldman-Rakic PS (1987) Circuitry of primate prefrontal cortex and regulation of behavior by representational memory. In: Handbook of physiology, the nervous system V (Plum F, Mountcastle V, eds), pp 373-417. Bethesda, MD: American Physiological Society.

Insausti R, Munoz M (2001) Cortical projections of the non-entorhinal hippocampal formation in the cynomolgus monkey (Macaca fascicularis). Eur J Neurosci 14:435-451.

Insausti R, Amaral DG, Cowan WM (1987a) The entorhinal cortex of the monkey. II. Cortical afferents. J Comp Neurol 264:356-395.

Insausti R, Amaral DG, Cowan WM (1987b) The entorhinal cortex of the monkey. III. Subcortical afferents. J Comp Neurol 264:396-408.

Ishii K, Kitagaki H, Kono M, Mori E (1996) Decreased medial temporal oxygen metabolism in Alzheimer's disease shown by PET. J Nucl Med 37:1159-1165.

Juottonen K, Laakso MP, Insausti R, Lehtovirta M, Pitkanen A, Partanen K, Soininen H (1998) Volumes of the entorhinal and perirhinal cortices in Alzheimer's disease. Neurobiol Aging 19:15-22.

Le Mestric C, Chavoix C, Miyazawa H, Tillet I, Allain P, Luet D, Gourand F, Fallet-Bianco C, Mézenge F, Brocquehaye A, Travère JM, MacKenzie ET, Baron JC (1993) Lesional models in the nonhuman primate brain: advances with magnetic resonance imaging (MRI) and coronal positron emission tomography (PET). In: Quantification of 
brain function: tracer kinetics and image analysis in brain PET (Uemura K, Lassen NA, Joues T, Kanno I, eds), pp 475-485. Amsterdam: Elsevier.

Le Mestric C, Chavoix C, Chapon F, Mézenge F, Epelbaum J, Baron JC (1998) Effects of damage to the basal forebrain on brain glucose utilization: a reevaluation using positron emission tomography in baboons with extensive unilateral excitotoxic lesion. J Cereb Blood Flow Metab 18:476-490.

Malkova L, Bachevalier J, Mishkin M, Saunders RC (2001) Neurotoxic lesions of perirhinal cortex impair visual recognition memory in rhesus monkeys. NeuroReport 12:1913-1917.

Meguro K, Blaizot X, Kondoh Y, Le Mestric C, Baron JC, Chavoix C (1999) Neocortical and hippocampal glucose hypometabolism following neurotoxic lesions of the entorhinal and perirhinal cortices in the non-human primate as shown by PET: implications for Alzheimer's disease. Brain 122:1519-1531.

Meguro K, Le Mestric C, Landeau B, Desgranges B, Eustache F, Baron JC (2001) Relations between hypometabolism in the posterior association neocortex and hippocampal atrophy in Alzheimer's disease: a PET/MRI correlative study. J Neurol Neurosurg Psychiatry 71:315-321.

Meltzer CC, Zubieta JK, Brandt J, Tune LE, Mayberg HS, Frost JJ (1996) Regional hypometabolism in Alzheimer's disease as measured by positron emission tomography after correction for effects of partial volume averaging. Neurology 47:454-461.

Meunier M, Bachevalier J, Mishkin M, Murray EA (1993) Effects on visual recognition of combined and separate ablations of the entorhinal and perirhinal cortex in rhesus monkey. J Neurosci 13:5418-5432.

Mielke R, Schroder R, Fink GR, Kessler J, Herholz K, Heiss WD (1996) Regional cerebral glucose metabolism and postmortem pathology in Alzheimer's disease. Acta Neuropathol (Berl) 91:174-179.

Murray EA (1996) What have ablation studies told us about the neural substrates of stimulus memory? Semin Neurosci 8:13-22.

O'Brien JT, Eagger S, Syed GM, Sahakian BJ, Levy R (1992) A study of regional cerebral blood flow and cognitive performance in Alzheimer's disease. J Neurol Neurosurg Psychiatry 55:1182-1187.
Ohnishi T, Hoshi H, Nagamachi S, Jinnouchi S, Flores LG, Futami S, Watanabe K (1995) High-resolution SPECT to assess hippocampal perfusion in neuropsychiatric diseases. J Nucl Med 36:1163-1169.

Perani D, Bressi S, Cappa F, Vallar G, Alberoni M, Grassi F, Caltagirone F, Cipolotti L, Franchi M, Lenzi GL, Fazio F (1993) Evidence of multiple memory systems in the human brain. Brain 116:903-919.

Rombouts SA, Barkhof F, Veltman DJ, Machielsen WC, Witter MP, Bierlaagh MA, Lazeron RH, Valk J, Scheltens P (2000) Functional MR imaging in Alzheimer's disease during memory encoding. AJNR Am J Neuroradiol 21:1869-1875.

Sabbagh MN, Lynn P, Jhingran S, Massman P, Villanueva-Meyer J, Olup J, Doody RS (1997) Correlations between SPECT regional cerebral blood flow and psychometric testing in patients with Alzheimer's disease. J Neuropsychiatry Clin Neurosci 9:68-74.

Soininen H, Helkala EL, Kuikka J, Hartikainen P, Lehtovirta M, Riekkinen PJS (1995) Regional cerebral blood flow measured by ${ }^{99 m}$ TcHMPAO SPECT differs in subgroups of Alzheimer's disease. J Neural Transm Park Dis Dement Sect 9:95-109.

Suzuki WA (1996) Neuroanatomy of the monkey entorhinal, perirhinal and parahippocampal cortices: organization of cortical inputs and interconnections with amygdala and striatum. Semin Neurosci 8:3-12.

Suzuki WA, Amaral DG (1994) Perirhinal and parahippocampal cortices of the macaque monkey: cortical afferents. J Comp Neurol 350:497-533.

Van Hoesen GW, Pandya DN (1975) Some connections of the entorhinal (area 28) and perirhinal (area 35) cortices of the rhesus monkey. I. Temporal lobe afferents. Brain Res 95:1-24.

Yamaguchi S, Meguro K, Itoh M, Hayasaka C, Shimada M, Yamazaki H (1997) Decreased cortical glucose metabolism correlated with hippocampal atrophy in Alzheimer's disease as shown by MRI and PET. J Neurol Neurosurg Psychiatry 62:596-600.

Zola-Morgan S, Squire LR (1985) Amnesia in monkeys after lesions of the mediodorsal nucleus of the thalamus. Ann Neurol 17:558-564. 